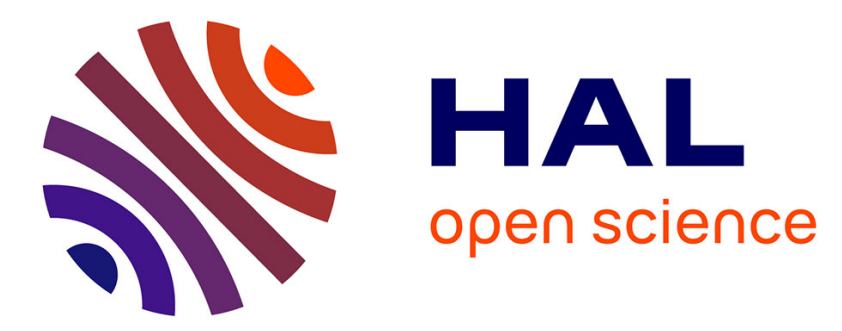

\title{
Numerical investigation of bend and torus flows, part I : effect of swirl motion on flow structure in U-bend
}

\author{
Jeremy Pruvost, J. Legrand, P. Legentilhomme
}

\section{To cite this version:}

Jeremy Pruvost, J. Legrand, P. Legentilhomme. Numerical investigation of bend and torus flows, part I: effect of swirl motion on flow structure in U-bend. Chemical Engineering Science, 2004, 59 (16), pp.3345-3357. 10.1016/j.ces.2004.03.040 . hal-02534046

\section{HAL Id: hal-02534046 \\ https://hal.science/hal-02534046}

Submitted on 17 Apr 2020

HAL is a multi-disciplinary open access archive for the deposit and dissemination of scientific research documents, whether they are published or not. The documents may come from teaching and research institutions in France or abroad, or from public or private research centers.
L'archive ouverte pluridisciplinaire HAL, est destinée au dépôt et à la diffusion de documents scientifiques de niveau recherche, publiés ou non, émanant des établissements d'enseignement et de recherche français ou étrangers, des laboratoires publics ou privés. 


\title{
Numerical investigation of bend and torus flows, part I : effect of swirl motion on flow structure in U-bend
}

\author{
J. Pruvost*, J. Legrand, P. Legentilhomme \\ GEPEA UMR-CNRS 6144, Faculté des Sciences et des Techniques, 2 rue de la Houssinière, BP 92208, 44322 Nantes, Cedex 3, France
}

Received 31 July 2002; received in revised form 7 November 2003; accepted 31 March 2004

\begin{abstract}
As a preliminary study to flow modeling in torus reactors, simulations are carried out in well-known standard geometries, namely $90^{\circ}$ and $180^{\circ}$ bends. For the latter, two flow configurations are considered, with and without initial swirl motion so as to approach torus reactor conditions. Efficiency of the commercial CFD code FLUENT is investigated by comparing predicted results with experimental measurements available in the literature for both bended configurations. Different turbulent models and near-wall considerations are considered, including $k-\varepsilon$ and high Reynolds-stresses models, with the standard wall-function approach as well as the two-layer zonal model and low-Re $k-\varepsilon$ models.

After validation of the numerical strategy, a parametric study is made to better understand the interactions between Dean vortices, involved by the bend curvature, and the main rotating motion generated by the swirl motion. Simulations are achieved for various values of the initial swirl intensity applied at the bend entry. Numerical simulations show different flow structures, resulting from the progressive Dean vortices perturbations with the increase of swirl intensity.
\end{abstract}

Keywords: Hydrodynamics; Numerical analysis; Bend; Simulation; Turbulence; Swirl

\section{Introduction}

Computational fluid dynamics (CFD) is useful for the design and the optimization of various processes. With the increase of computer resource and the enhancement of the efficiency of numerical algorithms, flows in complex geometries can now be studied using personal computers (PC). Reactors of torus shape are specific geometries because of their loop type and of the fluid circulation that occur by using an impeller. An efficient three-dimensional swirling motion is obtained, resulting from the combined effects of the rotation of the impeller and Dean vortices involved in reactor bends. But, despite the fact that efficient mixing conditions are achieved compared to classical stirred tanks (Belleville et al., 1992; Nouri et al., 1997; Legrand et al., 1997), only few investigations of the flow in torus reactors are available in the literature. Khalid et al. (1996) and Khalid and Legrand (2001) have emphasized the great influence of bends on velocity profiles, confirming that the resulting flow is explained

\footnotetext{
* Corresponding author. Tel.: +33-2-51-12-55-09; fax: +33-2-51-12-55-05.

E-mail address: jeremy.pruvost@physique.univ-nantes.fr (J. Pruvost).
}

by the coupling between bends effects and swirl motion involved by the impeller. This results in very complex flow conditions, making such geometries difficult to experimentally investigate, and thus, to analyze and optimize. For this purpose, CFD is a valuable tool, because it can provide access to various hydrodynamical characteristics in the entire geometry. Thus, a commercial code has been employed in this study for a numerical investigation of flow in torus reactor, and to provide a complementary and further characterization of particular hydrodynamical conditions involved in such geometries.

The complete modeling of the flow in the whole torus reactor is complicated, because of the bend curvature effects which have to be accurately represented, and of the impeller that needs to be modeled. To better understand the global resulting flow in the torus reactor, a first preliminary study is restricted to the modeling of flow through standard bends, with and without swirl motion applied in the bend entry. Bend flow results will allow a better understanding of the overall torus flow resulting from interactions between the bend curvature and the impeller rotation.

Bend flows have been extensively studied experimentally (Al-Rafai et al., 1990; Cheng and Farokhi, 1992; Kim and 
Simon, 1988), and numerically (Jayanti et al., 1990; Rütten et al., 2001; Van de Vosse et al., 1989; Wang and Shirazi, 2001; Bergstrom et al., 1998). It is well known that the flow in bends is affected by the wall curvature, which acts on the main features of the resulting flow, like mean velocity profiles, wall pressure and turbulent characteristics (Sudo et al., 1998; Cheng and Farokhi, 1992). For circular or square-sectioned ducts, the imbalance between the centrifugal force and the radial pressure gradient involved by the curvature leads to well-known Dean instabilities. A good numerical prediction of the flow downstream the bend requires an accurate representation of those instabilities (Lai et al., 1991; Ghia et al., 1987). To predict this secondary motion, the flow simulation has to be three-dimensional (Van de Vosse et al., 1989). Wang and Shirazi (2000) have investigated a two-dimensional square-sectioned bend, but the ratio of width to height of the channel was high enough to neglect the effects of Dean vortices.

Accuracy of numerical predictions depends on the bend curvature. Iacovides et al. (1996a) have shown that for bends with very strong curvature $\left(R_{c} / D=0.65\right)$, results are not satisfactory, even when very refined turbulence models are used. For bends with smaller curvature, better predictions are obtained, depending of the turbulence model employed. Iacovides et al. (1996b) have studied a square-sectioned $180^{\circ}$ bend with a bend curvature $R_{c} / D=3.35$, to emphasize the importance of the near-wall consideration. Indeed, it is well known that the turbulent boundary layer has to be accurately predicted in bends, because it directly affects the resulting core region flow (Lai et al., 1991). Iacovides et al. (1996a) have investigated the influence of the choice of the numerical strategies of resolution, by testing various wall considerations with different turbulence models. The aim of part I of this article is also to test numerical models for flow in circular-sectioned bends, with a more original part devoted to the effect of swirl motion on bend flows. Evaluation of the numerical approach accuracy will be enabled by comparison with data available in the literature.

Two test cases have been used for evaluation, one $90^{\circ}$ bend based on results of Sudo et al. (1998), and one $180^{\circ}$ bend studied by Anwer and So (1993). For the last case, experimental data were obtained with and without applying a swirl motion at the bend entry. Flow simulations have been obtained with the commercial code FLUENT.

\section{Description of test cases}

\subsection{The $90^{\circ}$ bend}

The investigated $90^{\circ}$ bend is described in Sudo et al. (1998). It consists of a circular pipe with an internal diameter $D=0.104 \mathrm{~m}$ and a curvature radius $R_{c}=0.208 \mathrm{~m}$, corresponding to a bend curvature $R_{c} / D=2$ (Fig. 1a). A fully-developed turbulent pipe flow is obtained at the entrance of the bend, with a mean bulk air velocity $U_{0}=$
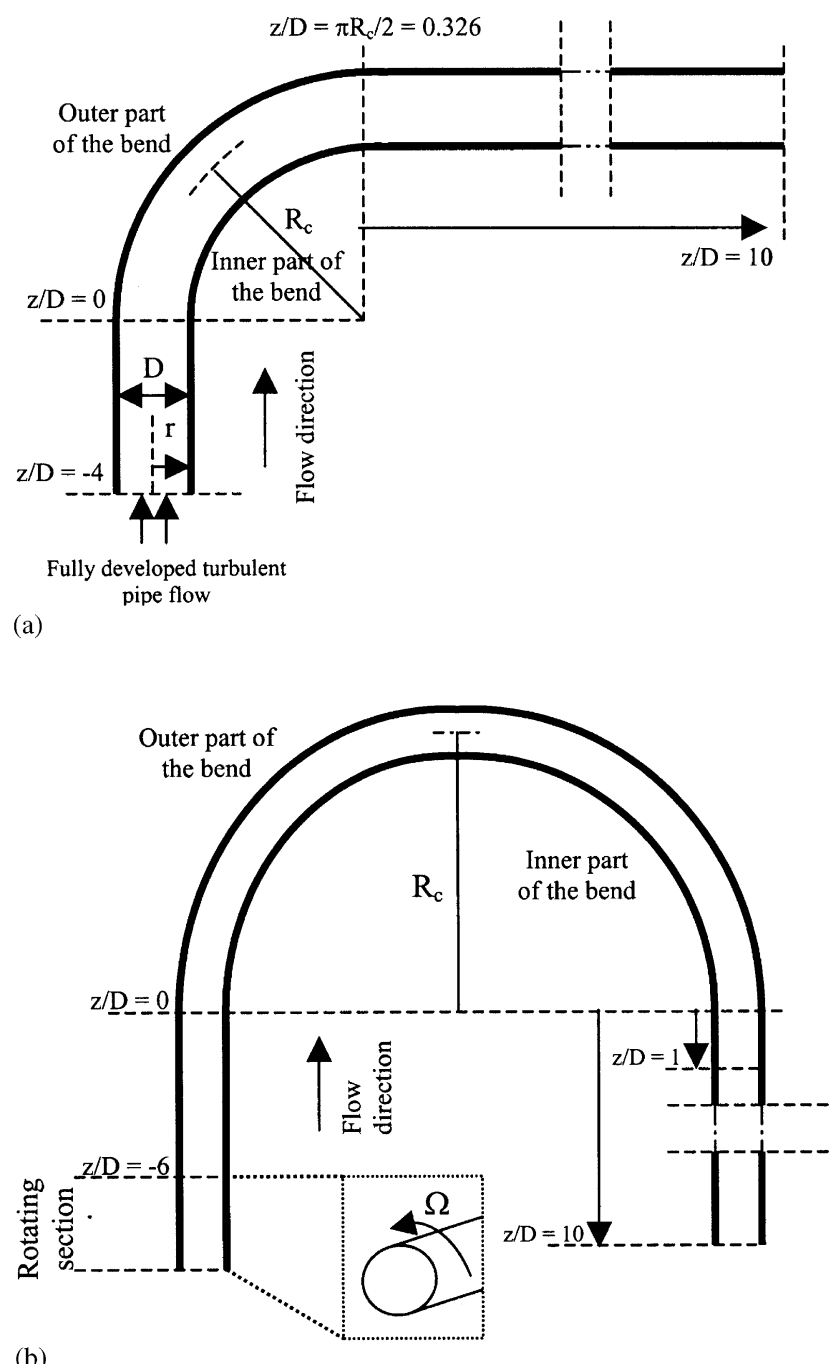

(b)

Fig. 1. Schematic representation of bends investigated: (a) $90^{\circ}$ bend; (b) $180^{\circ}$ bend.

$8.7 \mathrm{~m} \mathrm{~s}^{-1}$, corresponding to a Reynolds number $R e=60000$. The numerical modeling of the bend considers two straight parts, one upstream section with a length of 4 pipe diameters, and one downstream section of 10 pipe diameters to study the recovery of the flow after the bend.

\subsection{The $180^{\circ}$ bend}

The $180^{\circ}$ bend is described in Anwer and So (1993) and is shown in Fig. 1b. The internal diameter is $D=0.0762 \mathrm{~m}$ and the curvature radius $R_{c}=0.495 \mathrm{~m}$, leading to a bend curvature $R_{c} / D=6.49$. Two types of entrance conditions are investigated, one corresponding to a fully-developed turbulent pipe air flow, and one with an initial swirling motion superimposed to the main axial flow. The initial swirling flow is experimentally generated by a rotating section installed 6 diameters upstream of the curved bend entrance and sufficiently long to provide a complete solid-body rotation to 
the flow. The mean bulk velocity is kept constant for both hydrodynamical conditions, with a value $U_{0}=10 \mathrm{~m} \mathrm{~s}^{-1}$, corresponding to a Reynolds number $R e=50000$. For the modeling purpose, the upstream part of the bend will be a straight pipe with a total length corresponding to 6 diameters, and a straight pipe of 10 diameters will be considered downstream to the bend.

Following the definition of Gupta et al. (1984), the swirl number $S n$ is given by

$S n=\frac{\int_{0}^{R} \int_{0}^{2 \pi} U V r^{2} \mathrm{~d} r \mathrm{~d} \theta}{R \int_{0}^{R} \int_{0}^{2 \pi} U^{2} r \mathrm{~d} r \mathrm{~d} \theta}$,

where $U$ is the mean axial velocity component, $V$, the circumferential one, and $r$ and $\theta$ are, respectively, radial and angular coordinates referred to pipe center.

At the bend entry, the flow is in solid-body rotation (Anwer and So, 1993). The value of the initial swirl intensity can be approximated in terms of the mean angular velocity $\Omega$ of the rotating motion related to the mean bulk velocity $U_{0}$ :

$S n=\frac{\Omega R}{2 U_{0}}$.

The initial swirl motion obtained by Anwer and So (1993) is $S n=0.5$.

\section{Numerical method}

\subsection{Mesh consideration}

Both geometries are three-dimensionally meshed using the GAMBIT software (Fluent Inc.) with elementary hexahedral volumes. Meshes topologies are given in Fig. 2. For a given cross section, two zones are considered. A structured mesh is used in the circumferential part (zone 1 ), where wall effects require an accurate representation, while an unstructured mesh is employed in the bulk region where velocity gradients are less important. The discretization in the circumferential direction is uniform, but the radial distribution is chosen in order to increase the number of elementary volumes close to walls.

The grid is divided in three parts in the axial direction, the upstream part (A), the bend (B) and the downstream part (C). Mesh discretization in upstream and downstream parts are not uniform, so as to refine the cells density when going closer to the bend, the axial meshing following a uniform distribution in the bend (Fig. 2).

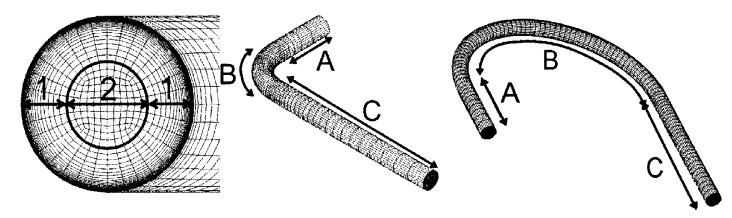

Fig. 2. Mesh topology of both bends.
Numerical resolutions will be made for two different near-wall considerations, one based on the application of the universal logarithmic velocity law (the wall-functions), and the second with a resolution of the momentum equations in the boundary layer using a two-layer zonal method or low-Reynolds $k-\varepsilon$ model.

One constraint of the grid generation is the position of the first elementary volume closest to the wall. A criterion $y^{+}$is usually defined to characterize this cell location in wall-coordinate:

$y^{+}=\frac{\rho u_{\tau} y}{\mu}$,

where $u_{\tau}$ is the wall-friction velocity, $y$, the normal distance from the center of the cell to the wall, $\rho$ the fluid density and $\mu$ the fluid viscosity.

When the logarithmic law is employed, $y^{+}$is usually set close to $y^{+}=30$ (Launder and Spalding, 1974) and, for the full boundary layer resolution, close to $y^{+}=1$ (Chang et al., 1995). For this last method, at least 10 cells in the boundary layer are needed. To define the boundary layer thickness, the criterion used in FLUENT is a wall-distance based turbulent Reynolds number $R e_{y}$. The boundary layer thickness is characterized by $R e_{y}<200$, where $R e_{y}$ is defined by

$R e_{y}=\frac{\rho \sqrt{k y}}{\mu}$,

where $k$ is the turbulent kinetic energy at the distance $y$ from the wall. In this two-zonal approach, boundary layer equations are applied for $R e_{y}<200$, and the turbulent model retained for the core flow is used for $R e_{y}>200$ (see "Turbulence models and near-wall consideration" for details).

Grid parameters were first estimated, and then adapted so as to follow the previous constraints after convergence. Detailed description of both final meshes are given in Table $1 \mathrm{a}$ for the $90^{\circ}$ bend and Table $1 \mathrm{~b}$ for the $180^{\circ}$ bend.

Grid independency of the results was verified by varying parameters of each original mesh defined in Table 1. Various tests were made by changing parameters of near-wall meshes as the first cell location, and the discretization step in the three axial, radial and circumferential directions. Influence of the grid was investigated by comparing the mean axial velocity and the turbulent kinetic energy profiles obtained in different positions in the bend. An example of results is given in Fig. 3 for each bend. Number of cells of the coarsen grids are 21840 for the grid used with wall-function approach $\left(90^{\circ}\right.$ bend), and 50244 with full boundary layer resolution $\left(180^{\circ}\right.$ bend $)$. Results are found independent of the numerical mesh for corresponding near-wall consideration. Finest grids presented in Table 1 have thus been retained for the numerical study.

\subsection{Turbulence models and near-wall consideration}

Turbulent flows are mainly numerically solved using methods based on the resolution of Reynolds time-averaged 
Table 1

Meshes discretization

\begin{tabular}{|c|c|c|c|c|c|}
\hline \multicolumn{3}{|l|}{ Wall-function approach } & \multicolumn{3}{|c|}{ Boundary layer full-resolution } \\
\hline \multicolumn{6}{|c|}{ (a) Number of cells for the $90^{\circ}$ bend } \\
\hline \multirow[t]{4}{*}{ Axial direction } & & 58 & Axial direction & & 58 \\
\hline & Zone A & 7 & & Zone A & 7 \\
\hline & Zone B & 25 & & Zone B & 25 \\
\hline & Zone C & 26 & & Zone $\mathrm{C}$ & 26 \\
\hline \multirow[t]{3}{*}{ Radial direction } & & 44 & Radial direction & & 68 \\
\hline & Zone 1 & 28 & & Zone 1 & 56 \\
\hline & Zone 2 & 16 & & Zone 2 & 12 \\
\hline \multirow{2}{*}{\multicolumn{2}{|c|}{$\begin{array}{l}\text { Circumferential direction } \\
\text { Total number of cells }\end{array}$}} & 32 & Circumferential direction & & 32 \\
\hline & & 39680 & Total number of cells & & 68706 \\
\hline \multicolumn{6}{|c|}{ (b) Number of cells for the $180^{\circ}$ bend } \\
\hline \multirow[t]{4}{*}{ Axial direction } & & 104 & Axial direction & & 104 \\
\hline & Zone A & 14 & & Zone A & 14 \\
\hline & Zone B & 60 & & Zone B & 60 \\
\hline & Zone C & 30 & & Zone $\mathrm{C}$ & 30 \\
\hline \multirow[t]{3}{*}{ Radial direction } & & 40 & Radial direction & & 64 \\
\hline & Zone 1 & 28 & & Zone 1 & 52 \\
\hline & Zone 2 & 12 & & Zone 2 & 12 \\
\hline Circumferential direction & & 32 & Circumferential direction & & 32 \\
\hline Total number of cells & & 59904 & Total number of cells & & 99840 \\
\hline
\end{tabular}

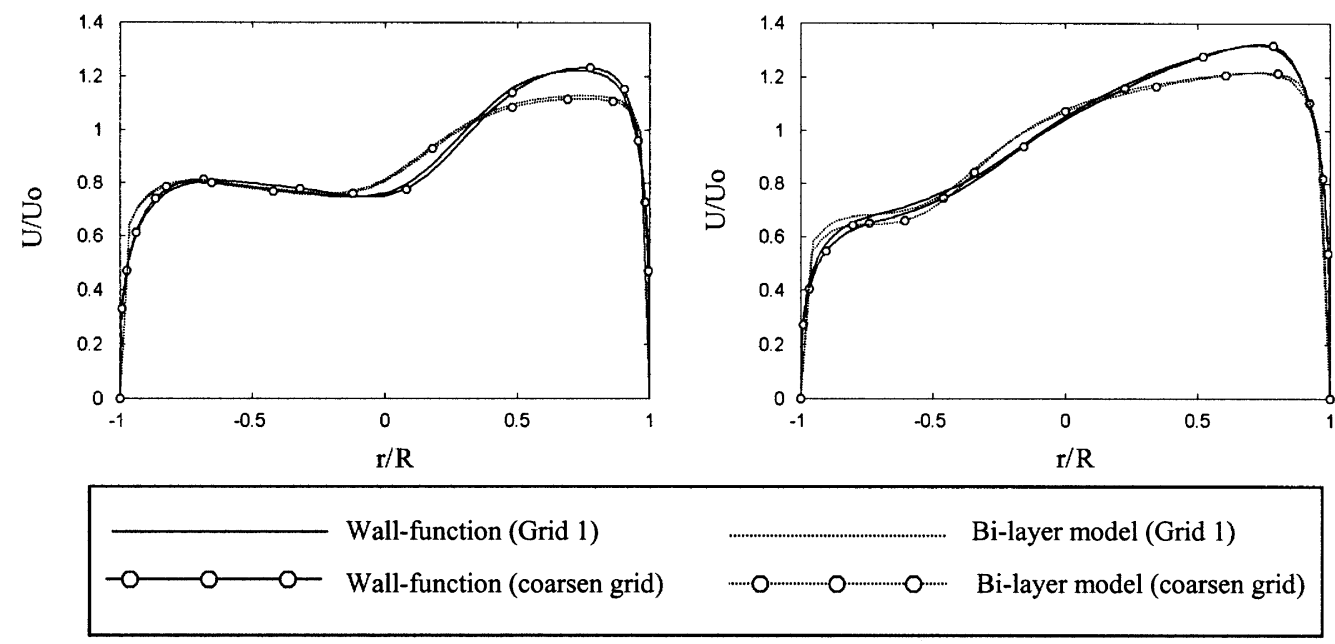

Fig. 3. Influence of the grid refinement using $k-\varepsilon$ model (left: mean axial velocity profile in the $90^{\circ}$ bend outlet; right: turbulent kinetic energy profile in the $180^{\circ}$ bend outlet).

Navier-Stokes (RANS) equations. Despite the fact that only average turbulent characteristics can be determined, this is often sufficient for the understanding of basic features of flows. Those turbulent models are divided in two categories depending on the modeling of turbulence stresses, based either on a eddy-viscosity model (EVM) or a Reynolds-stresses model (RSM). RSM are theoretically more adapted when turbulence is found to be anisotropic, as in bend flows (Lai et al., 1991).

The standard approach is to apply a wall-function based on the universal logarithmic profile (standard wall-function). But it has been demonstrated in many studies, and espe- cially for flows with streamlines curvature, that this simple consideration can remain inefficient because of the particular structure of the boundary layer that prevents the universal logarithmic profile to be applied (Lai et al., 1991; Kim and Rhode, 2000; Iacovides et al., 1996a). Various modified wall-functions are available in the literature. Kim and Rhode (2000) have studied the validity of the universal logarithmic law for flow in curved surfaces. They have emphasized the necessity of considering curvature effects in the wall-law, because of the perturbation of the boundary layer involved by extra strain-rate introduced by the bend curvature. They have proposed an adapted semi-empirical wall-function. 
Another solution consists in a full-resolution of the RANS equations in the boundary layer. Various wall models have been developed for this purpose, differences being in assumptions made about wall effects on the transport equations of the turbulence models in this particular region. Such approach can enhance the accuracy of the prediction but, as described before for the grid consideration, the drawback of solving the boundary layer momentum equations is the very fine grid requested in this region. It leads then to an increase in computational time. But, even in this approach, the near-wall turbulence model as to be retained. The theoretically best solution is to apply Reynolds-stress closure over the entire domain. For example, Iacovides et al. (1996a) have obtained an accurate prediction with a very complete model based on a resolution with Reynolds-stress closure, even in the boundary layer region. But, it must be noticed that the study of Iacovides et al. (1996a) deals with bend of very strong curvature of $R_{c} / D=0.65$. However, some examples are available in the literature for curvatures closer to those of both test cases retained in this study. Good predictions were achieved by using simple models. Wang and Shirazi (2001) have compared the standard $k-\varepsilon$ model with wall functions, and a low-Re $k-\varepsilon$ model, which solves the boundary layer momentum equations, to predict $90^{\circ}$ bend flow. Both approaches were in agreement with experimental data, and no significant improvement was observed with the boundary layer full-resolution, despite the simplicity of the association of standard $k-\varepsilon$ model with classical wall function. On the contrary, Lai et al. (1991) have used a Reynolds-stress closure in the boundary layer, and found a little improvement in prediction.

All those studies show that various numerical modeling solutions can be retained for bend flows, and predictions with more or less accuracy can be achieved. Furthermore, results obtained in curved pipes are difficult to extend to the case of swirling flows in bends, where hydrodynamical conditions are greatly modified by the swirl motion. Some numerical studies dealing with swirl flows are available, but only with straight pipes (Parchen and Steenbergen, 1998) or annular geometries (Farias Neto et al., 1998). Thus, regarding literature, a conclusion cannot be made about the more efficient model for the case under study.

As discussed before, the aim of this study was not to develop a particular model, but to predict swirl flows in bends, and next, flow in a torus reactor (see part II). The evaluation of numerical resolution efficiency will thus be made by testing different possibilities offered by the commercial code FLUENT, and especially combinations between various models and near-wall treatments. All the models available in FLUENT software $(k-\varepsilon$ based models, RSM, $k-\omega$ models) are well known and have been extensively used in CFD applications. Complete descriptions of those models can be easily found in the literature (Wilcox, 1998; Launder and Spalding, 1974), and only main features are thus presented here.
Models based on the $k-\varepsilon$ approach employ transport equations for the turbulence kinetic energy, $k$, and its dissipation rate, $\varepsilon$. The $k-\varepsilon$ RNG model is derived from the standard version of the $k-\varepsilon$ model, but is modified to improve predictions for transitional flows and flows presenting high streamline curvature. This model is thus theoretically more adapted for the prediction of the effect of swirl on turbulence. But, both $k-\varepsilon$ and $k-\varepsilon$ RNG models are of EVM type and use the Boussinesq hypothesis (Hinze, 1975), making turbulence to be considered as isotropic. If the flow is found to have a high anisotropic behavior, the Reynolds stress model is more suitable because of the calculation of the individual Reynolds stress. Another more recent EVM, the $k-$ $\omega$ model, is implemented in FLUENT software. The model used was proposed by Wilcox (1998), and is based on transport equations for the turbulence kinetic energy, $k$, and the specific dissipation rate, $\omega$, which can also be thought as the ratio of $\omega$ to $k$. This kind of model theoretically gives better prediction for low Reynolds number effects and shear flow spreading.

Turbulence models, including RSM, standard and RNG versions of the $k-\varepsilon$ model, are high-Reynolds-number models defined for turbulent core flows. As cited before, a wall consideration has to be used in conjunction with these models. The classical solution is to use wall functions. The drawback is the near-wall region which is not solved, but determined using semi-empirical formulae expressing the well-known log-law. FLUENT software offers possibilities to calculate near-wall flows, so as to achieve a better prediction of walls effects. The low-Re $k-\varepsilon$ approach considers these wall effects in its formulation, and thus can be used in regions where low Reynolds numbers are encountered, especially close to the wall. For this purpose, wall-distance based damping functions are added to the $\varepsilon$-transport equation and in the expression of the turbulent viscosity, in order to extend model transport equations in the boundary layer, including the viscous sublayer. Literature shows that accuracy of predictions depends on the choice of the damping functions, which are of semi-empirical nature and cannot consequently give accurate results in all types of flows. FLUENT provides variants of low-Re $k-$ $\varepsilon$ model, each one having an alternative form of damping functions. Only results achieved with the Chang-HsiehChen model, which was initially established for flow in pipe (Chang et al., 1995), are presented in this study, other models being less efficient. Another alternative solution is proposed for the near-wall consideration, the two-layer zonal model that solves the boundary layer. By using $R e_{y}$, a viscosity affected region is defined for $R e_{y}<200$, with a turbulent core region for $R e_{y}>200$ where classical turbulent model can be applied. In the viscosity-affected region, only the $k$-transport equation is solved, $\varepsilon$-transport equation being computed using a correlation involving a length scale (Wolfstein, 1969). This approach was tested in association with $k-\varepsilon$ models for the turbulent core flow. 
Finally, it must be noticed that FLUENT proposed other possibilities of modeling approaches. Various trials were made, but results are not presented in this paper because they are not adapted for the test cases, or no improvement was observed, like for example the use of the non-equilibrium wall function.

These following combinations have been employed in the present work:

(a) a standard $k-\varepsilon$ model with a standard wall-function,

(b) a $k-\varepsilon$ RNG model with a standard wall-function,

(c) a $k-\varepsilon$ RNG model with a two-layer zonal model, which allows resolution of the boundary layer equations,

(d) a low-Re $k-\varepsilon$ model, where the flow is solved in the entire domain with a standard $k-\varepsilon$ model, even in the boundary layer,

(e) a Reynolds-stress model with a standard wall-function.

Despite that the $k-\omega$ model has been tested for each case described next, same predictions than with the $k-\varepsilon$ model have been achieved. To clarify figures, results for this model are thus not presented.

\subsection{Boundary conditions and numerical details}

Inlet boundary conditions applied in bend entry are defined in terms of mean velocity, $k$ and $\varepsilon$. For non-swirling cases, those values are obtained by solving the fully developed turbulent flow achieved in a straight pipe for corresponding $R e$ and pipe diameter. For the swirling case with the $180^{\circ}$ bend, Anwer and So (1993) used a rotating duct in the bend entry. Inlet boundary conditions were thus defined by using curve fitting of the experimental data of Anwer and So (1993) measured at the outlet of the rotating section at $z / D=-4, z$ being the axial position with $z=0$ at the bend entry.

In FLUENT software, the governing integral equations for the conservation of mass and momentum are solved using a control-volume-based technique, with a segregated approach where equations are solved sequentially with implicit linearization. Volume-faces advective fluxes are approximated using a quadratic upwind interpolation "QUICK", that is more accurate for rotating flows. The pressure interpolation scheme was found to influence the numerical results, better predictions being achieved with "PRESTO" scheme, well-suited for the steep pressure gradients involved in swirling flows. The pressure-velocity coupling is solved using the iterative correction procedure "SIMPLEC". Finally, the resulting linear set of equations is solved with an implicit linear equation solver (Gauss-Seidel) in conjunction with an algebraic multigrid method (AMG). Computations are performed by iterating until convergence (no variation of all convergence criteria associated to the set of governing equations). For all simulations, default constants of the turbulent models retained in FLUENT are employed and a steady-state calculation is made.

\section{Bend flows simulations}

\section{1. $90^{\circ}$ bend flow}

The $90^{\circ}$ bend is investigated to make a first estimation of the effectiveness of the different turbulence models. Various numerical approaches are evaluated by determining axial mean velocity profiles, $U$, obtained at the outlet of the bend $\left(z / D=\pi R_{c} / 2=0.326\right)$, and the longitudinal evolution of the wall-pressure coefficient, $C_{p}$, determined, respectively, for the inner and outer parts of the bend, where $C_{p}$ is

$C_{p}=\frac{\left(P-P_{\text {ref }}\right)}{\rho U_{0}^{2}}$,

with $P$, the wall static pressure and $P_{\text {ref }}$ the static pressure at $z / D=-4$.

Predictions obtained by different turbulence models are provided in Fig. 4. Except when specified, the profiles given along the radial coordinate are located in the symmetrical plane of the bend. No great difference is observed for the majority of models, good prediction being generally achieved. Solving the boundary layer equations leads to a little improvement. But, the use of the standard wall function, despite its simplicity when applied in the case of a bend, leads to a good prediction, showing the robustness of the method. Results are almost equivalent to those of the low-Re $k-$ $\varepsilon$ model. This was already observed by Wang and Shirazi (2001), which employed both methods in a similar geometry.

In conclusion of the $90^{\circ}$ bend flow study, good predictions are achieved for almost all the models tested. The shift of the maximum velocity toward the outer part of the bend is well predicted, showing an accurate representation of the effect of the centrifugal force induced by the bend curvature on hydrodynamical characteristics. In the same way, wall pressure are in agreement with experimental measurements. The increase of pressure on the outer part of the bend, and its decrease on the inner part are correctly predicted, as well as the wall-pressure recovery after the bend.

\section{2. $180^{\circ}$ bend flow without swirl motion}

Fig. 5 depicts the results obtained for the $180^{\circ}$ bend without initial swirl motion. Comparison has been made at the bend outlet $(z / D=1)$ for the axial component of the mean velocity, $U$, and for the turbulent kinetic energy profile, $k$. Wall friction velocity value, $u_{\tau}$, used for calculation of $k / u_{\tau}^{2}$, is the constant value $u_{\tau}=0.323 \mathrm{~m} \mathrm{~s}^{-1}$ obtained experimentally at $z / D=-18$ by Anwer and So (1993) in the fully developed turbulent pipe flow region.

For the mean velocity, no great difference between model predictions is observed and, as for the $90^{\circ}$ bend, experimental measurements are almost well-predicted. The centrifugal effect of the bend, which shifts the maximum of the axial velocity toward the outer wall is well represented. But, unlike for the $90^{\circ}$ bend, it can be noticed that accuracy decreases 

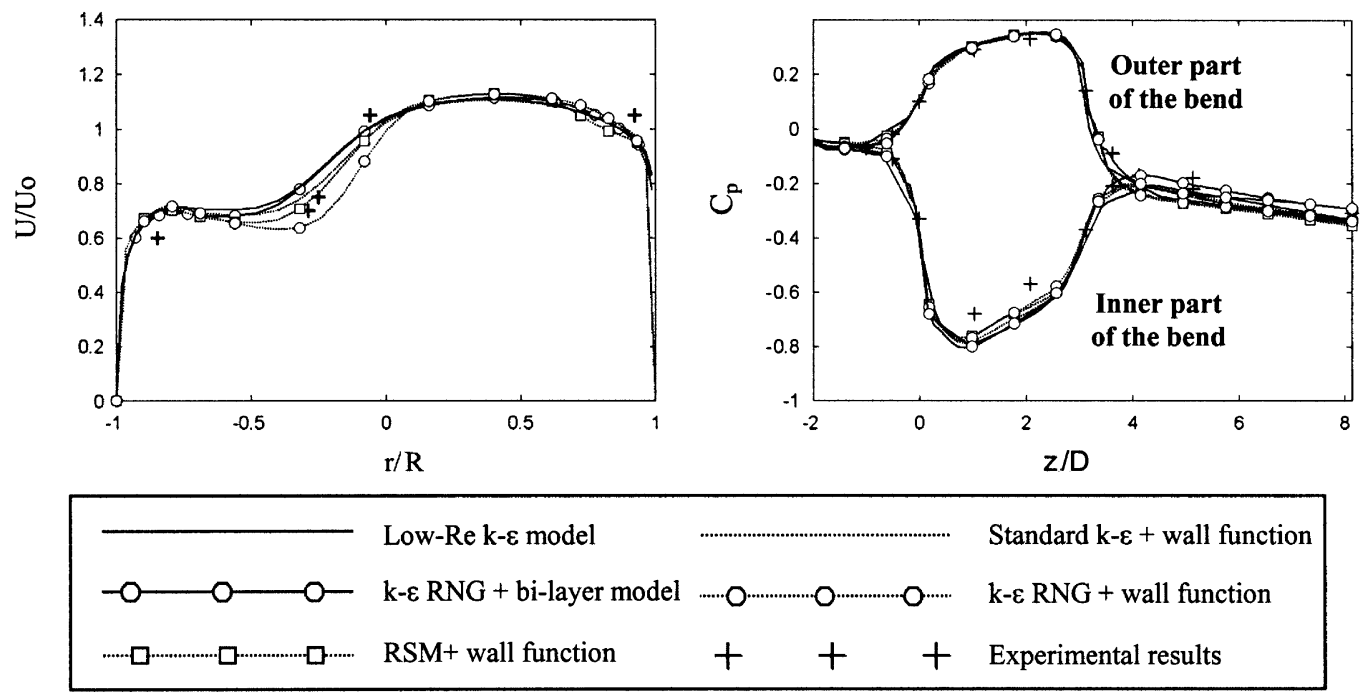

Fig. 4. Results of different numerical models for the $90^{\circ}$ bend (left: mean axial velocity profile at the bend outlet; right: longitudinal distribution of wall pressure coefficient).
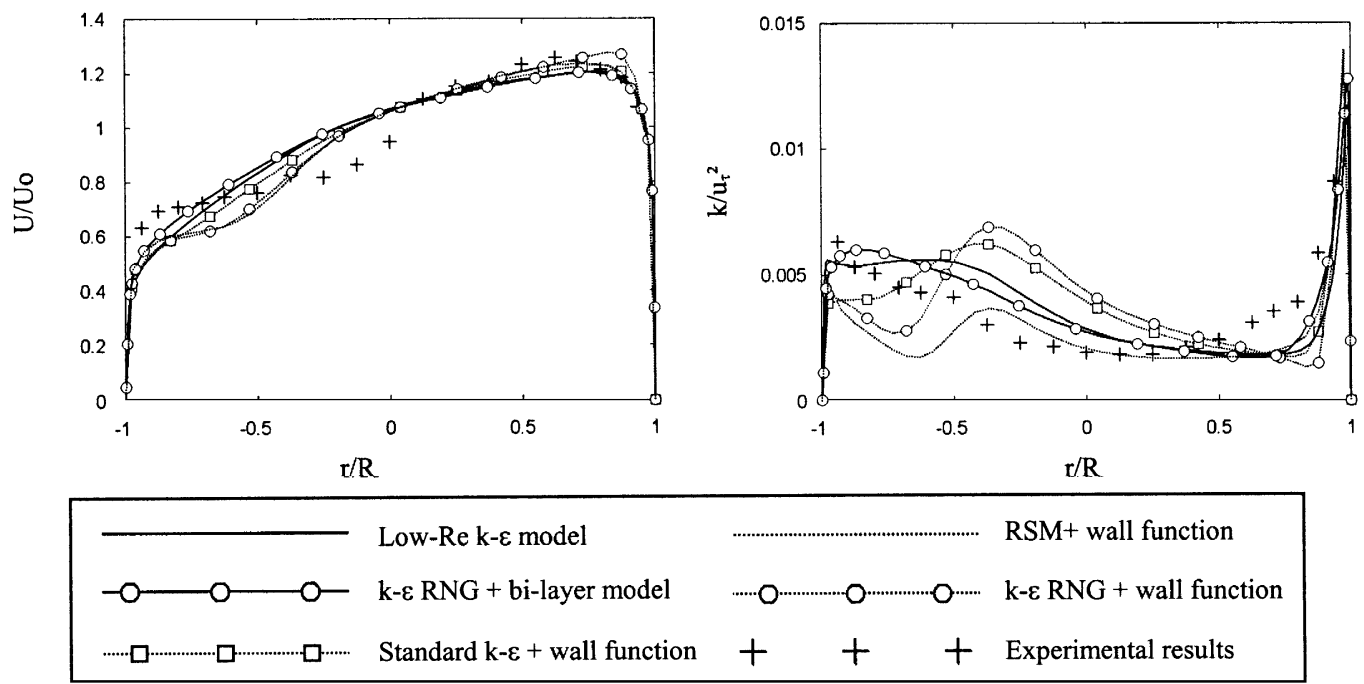

Fig. 5. Results of different numerical models for the $180^{\circ}$ bend at $z / D=1$, without swirl motion (left: mean velocity profile; right: turbulent energy profile).

significantly at the inner part of the $180^{\circ}$ bend. This shows the difficulty in predicting bend curvature effects. If the results obtained with the different turbulence models are compared in this region, no influence is observed, $k-\varepsilon$ RNG and RSM models giving almost the same predictions, despite turbulence anisotropy consideration involved in RSM models. Differences on mean velocity profile prediction appear to be mainly explained by the choice of the wall-consideration. Greater values are obtained in the inner part of the bend with a complete resolution of the boundary layer using a two-zonal approach or the low-Re $k-\varepsilon$ model. For the core region and the outer part of the bend, differences in predictions between models vanish. But, comparison on mean velocity profiles with experimental results cannot allow a full validation of one of the approach, considering uncertainty on experimental data.
If the turbulent kinetic energy profile is considered, a fairly good representation of the bend curvature effect is observed, with a lower turbulent kinetic energy level in the inner part of the bend, where the curvature greatly modifies the resulting turbulent kinetic energy distribution, as confirmed by Cheng and Farokhi (1992). Contrary to the mean axial velocity profiles, differences exist between the turbulence models for the turbulence characteristics predictions, as already observed by Wang and Shirazi (2000). Those authors have found that, even if no differences were obtained for mean values, various turbulent kinetic energy profiles were observed depending on the models retained. In the inner part of the bend under study, poor predictions are achieved using wall-function approach, whatever the turbulence model. Turbulent kinetic energy is underestimated in the near-wall region, and then overestimated when approaching the core 

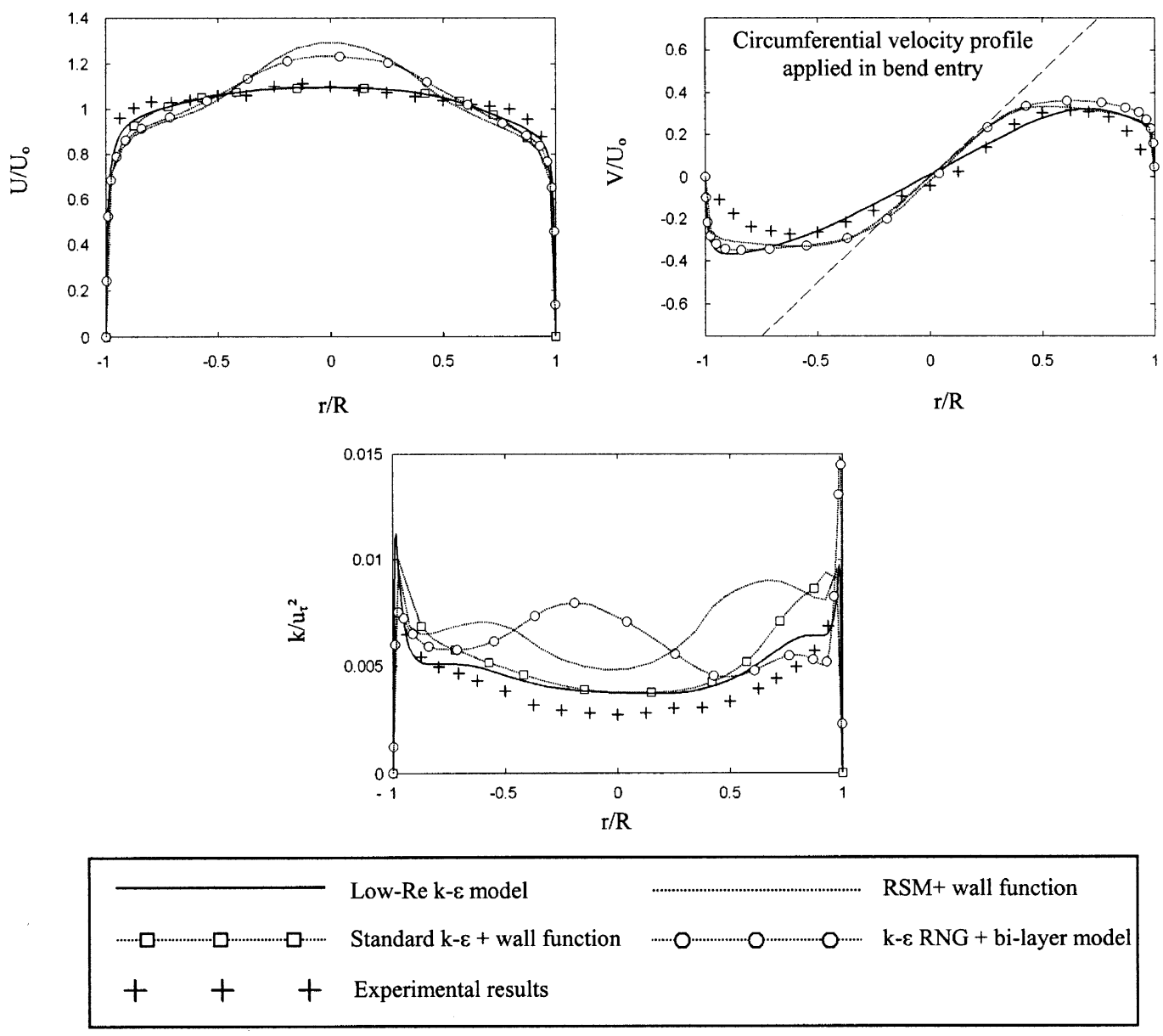

Fig. 6. Results of different numerical approaches for the $180^{\circ}$ bend at $z / D=1$, with $S n=0.5$ (left: mean axial velocity profile; right: mean circumferential velocity profile; bottom: turbulent energy profile).

flow region, to finally provide the same results than those obtained with a full resolution of boundary layer equations in the outer part of the bend. This last method leads to better results in the inner part of the bend, emphasizing the importance of the resolution of boundary layer equations to capture good features of the core flow behavior. Due to the bend curvature, wall-function approaches are less accurate, because the velocity distribution in the boundary layer deviates from the logarithmic assumption. With the full resolution of this region, the boundary layer is better predicted.

\section{3. $180^{\circ}$ bend flow with swirl motion}

Relevant results of numerical predictions are compared in Fig. 6 for the mean axial velocity profile $U$, turbulent kinetic energy $k$ and for the mean circumferential component obtained in the bend outlet, $V$.

If the mean axial velocity is considered, profiles of different shapes are obtained, especially in the pipe center region. Experimentally, a flat-type profile is measured, the shift of the maximum of the axial velocity towards the outer bend being not observed with swirl motion. This fact is explained by the swirl motion which dominates the flow, and thus decreases the curvature influence. The low-Re $k-\varepsilon$ model gives the better prediction, in good agreement with experimental data, but it can be observed that the standard version of the $k-\varepsilon$ model gives similar results, except near the walls where the association with the wall-function reduces the prediction accuracy. Great differences appear with other modeling approaches and, unlike without swirl motion, the choice of the turbulence model is more crucial in this case. No suitable predictions are obtained with either the high-Re RSM and the $k-\varepsilon$ RNG model, despite the fact that the $k-\varepsilon$ RNG model is theoretically a standard $k-\varepsilon$ model improved for swirling flows. This shows the inadequacy of the $k-\varepsilon$ RNG model to predict the turbulence in the core flow, as already observed by Parchen and Steenbergen (1998) who have compared a standard $k$ $-\varepsilon$ model with a modified version for swirling flow simulation. The free decay of swirl was better predicted using the simpler model.

To explain differences between numerical predictions, the logarithmic formulation of the velocity $u^{+}$in function of the 

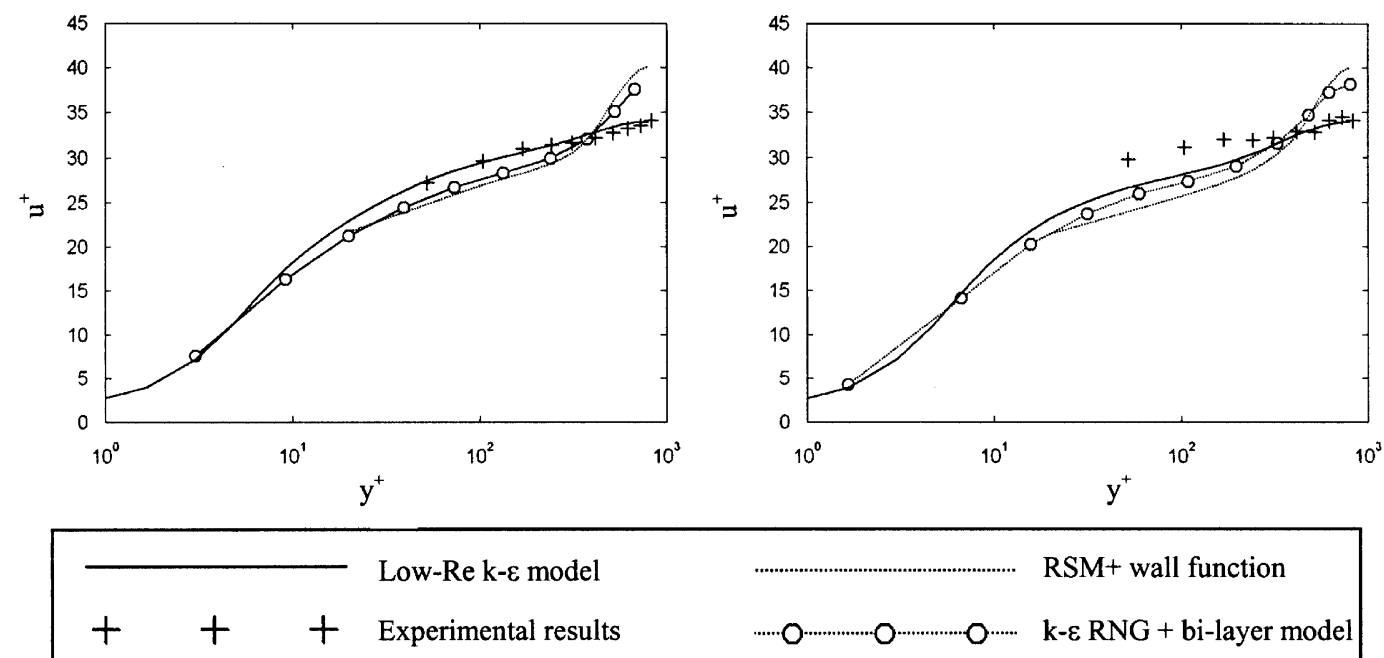

RSM+ wall function

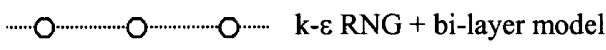

Fig. 7. Mean axial velocity predictions using wall coordinates at $z / D=1$ (left: outer part of the bend; right: inner part of the bend).

wall coordinate $y^{+}$is calculated, with

$u^{+}=\frac{U}{u_{\tau}}$.

When a near-wall function is used, the velocity between the wall and the first elementary volume is assumed to follow a logarithmic profile. Fig. 7 shows the inaccurate prediction of the near-wall velocity with this assumption, value obtained in the first elementary volume being underestimated. This confirms Kim and Rhode (2000) remarks, who have compared various wall-functions to experimental measurements in the boundary layer. For the outer region of the bend, predictions are more accurate than for the inner part, especially for the low-Re $k-\varepsilon$ model which agrees well with experimental data for the outer part, the two-layer zonal model being less accurate, but better than the wall-function approach. This shows the interest of a good prediction of the boundary layer. By allowing a more accurate representation of the boundary layer, the low-Re $k-\varepsilon$ model gives correct results in the core flow, as already observed in Fig. 6, despite the relative approximated hypothesis implied in the use of a turbulent EVM for swirling flows. For wall-function, due to bend curvature, velocity profile deviates from that on flat walls and the universal logarithmic law is not verified. This can explain the inefficiency of RSM. Despite turbulence anisotropy consideration in RSM that theoretically allows better prediction of swirling flows, a wrong representation of the boundary layer leads to an inaccurate prediction of the whole flow.

By considering the predictions of mean circumferential velocity profiles in bend (Fig. 6), the decrease of the swirl motion is relatively accurately predicted by the numerical simulation. The initial circumferential profile applied at the bend entry is added in the figure (dashed line). It corresponds to a forced-vortex type flow, as obtained by Anwer and So (1993). After crossing the bend, a free-vortex part appears in the vicinity of the walls, with a forced-vortex part remaining in the core flow region, between $r / R=-0.5$ and $r / R=0.5$. This feature is characteristic of free decaying swirling flow in pipes, and was already observed by many authors (Gupta et al., 1984; Clayton and Morsi, 1984; Farias Neto et al., 1998; Parchen and Steenbergen, 1998; Steenbergen and Voskamp, 1998). No significant differences are observed with the various numerical models, except for the low-Re and the standard $k-\varepsilon$ models which better predict the experimental data.

Turbulent kinetic energy profiles emphasize main conclusions observed by analyzing the mean axial velocity. The better prediction is achieved using the low-Re model which agrees with experimental data, other models giving different profiles. In the same way as without swirl motion, the near-wall resolution appears to influence the resulting evolution in the core region, especially for the outer part of the bend where only full resolutions of the boundary layer equations lead to a good approximation of the near-wall evolution of the turbulent kinetic energy profile, for both two-layer zonal and low-Re $k-\varepsilon$ models. With the wall-function approaches, turbulent kinetic energy values are over-estimated, and corresponding predictions in the core flow are not accurate compared to experimental data. It can be observed that, as with the mean axial velocity profile, no accurate prediction is achieved with the $k-\varepsilon$ RNG model despite its association with the two-zonal model, showing one more time its inadequacy for the flow under study. For the RSM, a more accurate representation is obtained in the core region, but because near-wall regions are not well-represented due to the use of a wall-function approach, the final prediction is not correct. Finally, the $k-\varepsilon$ standard model again gives satisfactory results in the core flow, despite its simple formulation, showing the robustness of its implementation in FLUENT software. Predictions in the wall-region are, however, less accurate than that obtained using the low-Re model. 
In conclusion, the accuracy of the numerical prediction of the bend swirling flow depends on the choice of the turbulent models. The $k-\varepsilon \mathrm{RNG}$ model is found to be inappropriate, and the high-Re RSM gives better results for the turbulent kinetic energy profile. But, because of association with a wall-function approach, this last model is not fully effective. Considering different possibilities offered by the FLUENT code, the Chang-Hsieh-Chen low-Re $k-\varepsilon$ model has to be preferred. Despite the fact that hydrodynamical conditions in a swirling flow are known to be far from the hypothesis assumed in the EVM, resulting mean and turbulent features of the flow are well-predicted due to a good resolution of the particular behavior of the bend boundary layers. Other models give satisfactory results, especially the standard $k-$ $\varepsilon$ one, despite its simplicity.

\section{Investigation of the influence of the swirl intensity on the bend flow}

To characterize the evolution of the flow as a function of the initial swirl intensity applied in bend entry, calculations are made for swirl number values ranging between 0 and 0.5. For these values, the low-Re $k-\varepsilon$ model is found to be accurate. An additional swirl number of 2.5 will be considered. Lack of experimental data does not allow the validation of the predictions for so high values of the swirl intensity, but results are of qualitative interest to illustrate the flow behavior in the bend when motion is swirl-dominated.

For boundary values of the mean velocity components, axial and radial velocities were the same than that obtained by Anwer and So (1993) for $S n=0.5$. The swirl intensity has a negligible influence on velocity boundary values, as it is shown for flow in a straight pipe with various swirl intensities (Anwer and So, 1989). For the circumferential velocity, values were determined so as to give a swirl motion of forced-type, with intensity corresponding to different swirl numbers investigated. To determine initial values of the turbulent kinetic energy and of the turbulent dissipation rate that are not experimentally available, different simulations of fully-developed swirling turbulent flows in a straight pipe were carried out (Anwer and So, 1989). Swirling flows are obtained by applying to the pipe wall an angular rotation velocity $\Omega$ corresponding to the swirl number investigated (Eq. (2)). Turbulent characteristics achieved in the straight pipe outlet were used as inlet conditions for the bend. A good agreement was observed between values obtained numerically for $S n=0.5$ and Anwer and So (1993) experimental data.

Velocity profiles for the axial mean velocity and turbulent kinetic energy in the bend outlet are shown in Fig. 8. A decrease of the bend curvature effect with the increase of the swirl number is observed. For $S n=0.125$, the maximum of axial velocity shifts towards the axis of the bend, with respect to the case of $S n=0$, to be finally located at about
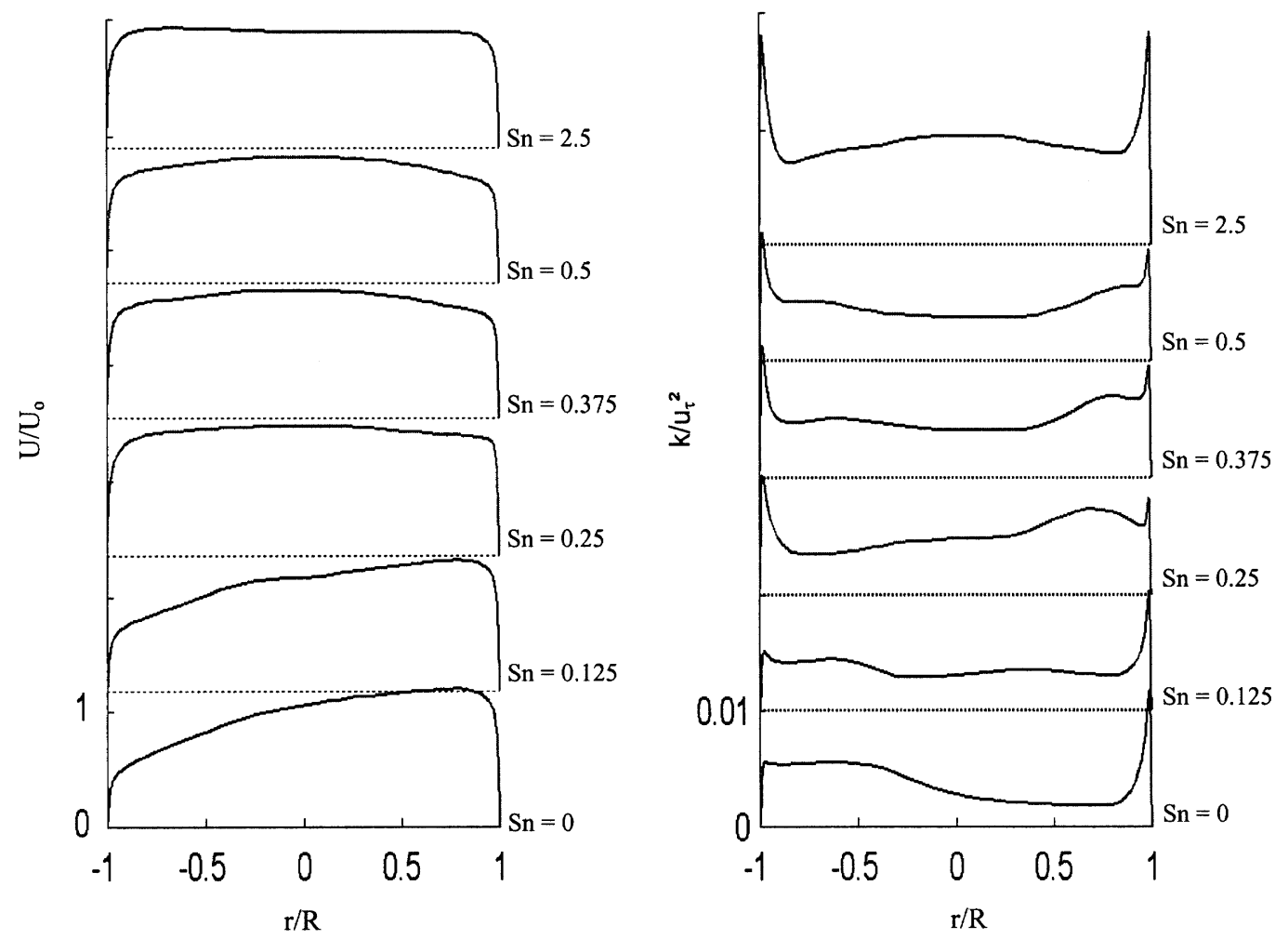

Fig. 8. Evolution of hydrodynamical characteristics at $z / D=1$ as a function of initial swirl number (left: mean axial velocity; right: turbulent kinetic energy). 


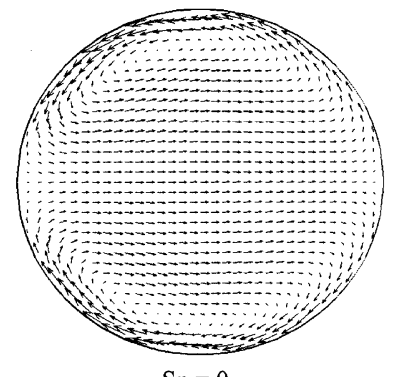

$\mathrm{Sn}=0$

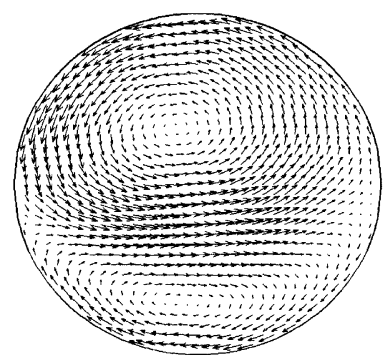

$\mathrm{Sn}=0.25$

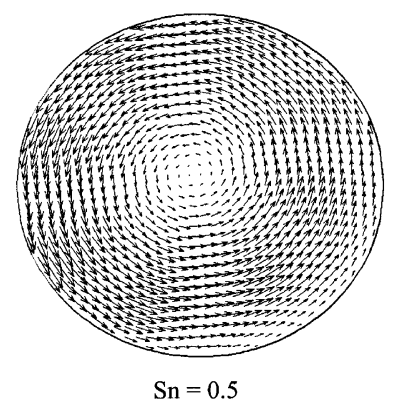

Fig. 9. Flow structure in a pipe section located at $z / D=1$.

the bend center for greater values of swirl intensity. The axial velocity profile is quasi-flat for $S n=2.5$. By applying a swirl motion at the entrance of the bend, curvature effects decrease.

Turbulent kinetic energy profiles confirm the remarks made for the mean axial component. Without swirl motion, the bend curvature leads to high values of turbulent kinetic energy in the outer part of the bend, while values in the inner part are kept low. The resulting profile is thus not symmetric with respect to the pipe center. But, if a swirl motion is applied, this symmetry tends to be restored, the flow finally becoming fully axisymmetric at $S n=2.5$. Compared to mean profiles, turbulent values appear to be more sensitive to the bend curvature, indeed a slight influence of the curvature can still be observed for $S n=0.5$. But, as for the axial mean velocity, a sudden evolution appears when increasing $S n$ from 0.125 to 0.25 . To explain this feature, the flow structure was represented in the bend outlet by plotting corresponding secondary velocity-fields in the pipe section. Results are given for various swirl numbers in Fig. 9. Without swirl motion, classical symmetrical and counter-rotating
Dean vortices generated by the bend curvature are shown. They are located in the upper and lower parts of the pipe section. If the velocity-field obtained for $S n=0.125$ is considered, despite the low swirl intensity, an evident perturbation of the flow symmetry of Dean vortices appears. Both vortices move towards the pipe center, the lower one being insensitive to swirl effect and better structured than the upper one. This is explained by the swirl motion, which tends to give a solid-body rotating motion to the flow. Because Dean vortices are counter-rotating, each one differently interacts with the swirl motion, leading to a resulting perturbation of the initial symmetric flow structure and Dean vortices locations. This emphasizes the competition between swirl motion and Dean vortices. This feature is more evident for $S n=0.25$. The resulting flow appears to be better structured, but with a different organization from the one obtained without swirl motion. The two counter-rotating structures are at different locations and with different intensities. For $S n=0.25$, the upper structure is of greater intensity and rotates in the same angular direction as the applied swirl motion. This vortex is thus not only caused by the bend curvature, but mainly due to the rotating motion involved by the swirl motion. This is confirmed by the result obtained for $S n=0.375$. Only one structure remains, approximately located at the center of the pipe. The bottom structure has vanished, and a unique global rotating motion is observed, filling all the pipe section. The flow at the bend outlet is thus swirl-dominated, and the high swirl intensity prevents from Dean vortices appearance. For $S n=0.5$, a same flow structure is observed, the only difference being in the shift of the center of the vortex towards the pipe center.

Instabilities of Dean-type are caused by the imbalance that arises in bends between centrifugal force and radial pressure gradient. When a swirl motion is added, both effects are modified. Results show that both phenomena greatly interact for values of swirl intensity up to $S n=0.25$. Then, by increasing the initial swirl intensity, the flow becomes suddenly swirl-dominated, with only one main rotating motion, to be finally only slightly modified by the bend curvature, as shown for $S n=2.5$.

To characterize bend curvature effects on the swirl motion, the resulting swirl number has been calculated at the inlet and outlet sections of the bend for each initial swirl intensity. Results were obtained by integrating numerically mean axial and circumferential velocity distributions in Eq. (1). A swirl intensity drop criterion $S_{1}$ was defined as follows:

$S_{1}=\frac{S n_{i}-S n_{o}}{S n_{i}}$,

where $S n_{i}$, is the swirl number at the bend inlet, and $S n_{o}$, at the outlet.

Results obtained in the bend were compared with $S_{1}$ values determined numerically in a straight pipe having same corresponding length as the bend investigated. To obtain these results, same boundary conditions were applied at the pipe entry, and the evolution of $S_{1}$ was determined by solving the 


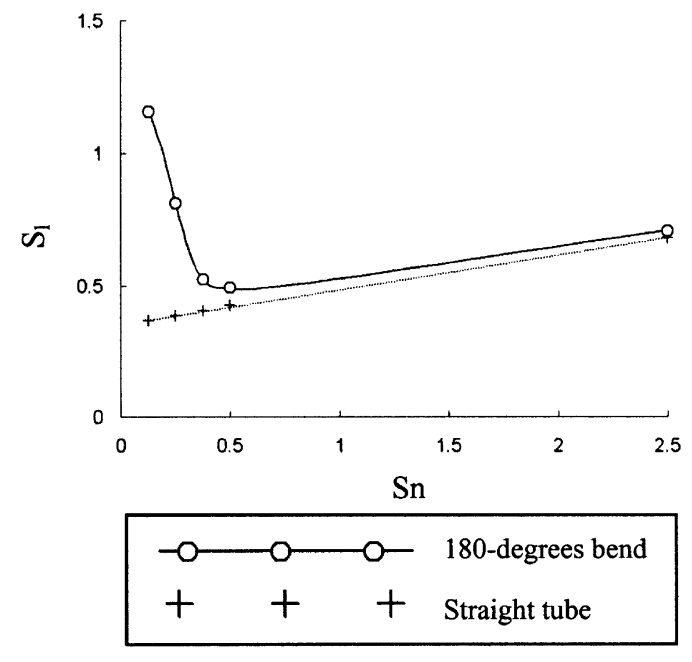

Fig. 10. Swirl intensity decrease criterion, $S_{1}$, evolution as a function of initial swirl number.

swirl free decay in the pipe. Results are given in Fig. 10, as a function of the initial swirl number applied at the entry.

It can be observed that the relative decrease of swirl intensity $S_{1}$ when crossing the bend is greater for low initial values of the swirl number. This confirms the competition between the swirl flow and Dean vortices which tends to disturb the swirl rotating motion. Furthermore, a value of $S_{1}$ greater than one is observed for the smallest swirl number. This means that a negative value of the swirl number is obtained at the outlet. Positive swirl number $\mathrm{Sn}_{o}$ is defined as rotating in the same direction as the main swirl motion. This negative value of $S_{1}$ means that Dean vortices have a preponderant influence, leading to a rotating motion in the opposite direction of the swirl motion. The resulting flow is thus the sum of Dean vortices and of the swirl motion, with a mainly Dean vortex driven flow. Then, $S_{1}$ decreases with increasing initial swirl number, until a threshold value of $S n=0.25-0.375$ is reached. Next, for greater values of $S n, S_{1}$ starts to increase. This agrees with previous remarks about the sudden evolution of flow characteristics observed with velocity-fields. For higher values of $S n$, the flow is swirl-dominated, that prevent appearance of Dean vortices. The bend curvature has only a slight influence, and the swirl flow decay when crossing the bend is almost similar as that obtained in a straight pipe of similar length, as shown in Fig. 10.

\section{Conclusion}

The numerical flow prediction in two different bends was investigated using the commercial CFD code FLUENT. The results establish the effectiveness of the code to determine flow conditions for both $90^{\circ}$ and $180^{\circ}$ bends. Different modeling approaches were tested, with various turbulence models and three types of near-wall modeling strategies, using a wall-function, a two-layer zonal model or a low-Re $k-\varepsilon$ model with boundary layer resolution. Without swirl motion, they were no great differences in the predictions of the various turbulence models. A slight improvement was observed with low-Re $k-\varepsilon$ models but the standard version of the $k-\varepsilon$ model revealed accurate.

The study has been next extended to the case of a bend flow with initial swirl motion. Results show the need of solving the boundary layer to well predict flow at the outlet. The choice of the turbulence model is found to be important too. If the boundary layer is not correctly determined, the resulting final prediction is less accurate. Despite its EVM approach which prevents it from taking into account the anisotropy of the flow turbulence, the low-Re $k-\varepsilon$ model is the most efficient, especially for the turbulence quantities, because it allows a good prediction of the boundary layer. Comparison of this model to experimental data validates the method for a swirl number of 0.5. But, even in this more complex case, acceptable predictions were achieved with the simple approach using the $k-\varepsilon$ model and the wall-function, showing the robustness of this association using FLUENT software. Because of the very fine grid requested for the low-Re $k-\varepsilon$ model, such a solution appears as an interesting alternative of modeling of swirling flows in bends by using a coarsen grid. Main features like mean velocities are predicted with sufficient accuracy.

Finally, to better understand the swirl effect in a bend flow, additional simulations were performed for different values of the swirl number applied at the bend entry. The competition between Dean vortices and swirl was emphasized, linked to the perturbation by the swirl motion of the radial pressure gradient due to the bend curvature. Three different cases of flow were observed as a function of swirl intensity. For small values of $S n$, the flow at the bend outlet was found to result from a complex interaction between Dean vortices and swirl motion. For higher values, influence of the swirl motion increased until a sudden variation was observed for $S n$ values ranged between 0.2 and 0.3 , corresponding to the Dean vortices disappearance. A further increase of $S n$ leads to a swirl dominated flow, with only poor influence of the bend curvature.

\section{Notation}

$C_{p} \quad$ wall pressure coefficient

$D$ pipe diameter, $\mathrm{m}$

$k$ turbulent kinetic energy, $\mathrm{m}^{2} \mathrm{~s}^{-2}$

$r$ radial coordinate, $\mathrm{m}$

$R \quad$ pipe radius, $\mathrm{m}$

$R_{c} \quad$ bend curvature radius, $\mathrm{m}$

Re Reynolds number $=\rho U_{0} D / \mu$

$R e_{y}$ wall-distance based turbulent Reynolds number

$S_{1}$ swirl intensity drop criterion

$S n$ swirl number

$u_{\tau} \quad$ wall friction velocity, $\mathrm{m} \mathrm{s}^{-1}$ 
$u^{+} \quad$ axial velocity in wall coordinate

$U$ mean axial velocity component, $\mathrm{m} \mathrm{s}^{-1}$

$U_{0} \quad$ mean bulk velocity, $\mathrm{m} \mathrm{s}^{-1}$

$V$ mean circumferential velocity component, $\mathrm{m} \mathrm{s}^{-1}$

$y \quad$ wall distance

$y^{+} \quad$ wall distance in wall coordinate

$z \quad$ longitudinal coordinate, $\mathrm{m}$

\section{Greek letters}

$\varepsilon \quad$ turbulent dissipation rate, $\mathrm{m}^{2} \mathrm{~s}^{-3}$

$\theta \quad$ angular coordinate, rad

$\mu$ dynamic viscosity, $\mathrm{Pa} \mathrm{s}$

$\rho$ fluid density, $\mathrm{kg} \mathrm{m}^{-3}$

$\Omega \quad$ angular velocity of the rotating section in the $180^{\circ}$ bend entry, $\operatorname{rad~s}^{-1}$

\section{References}

Al-Rafai, W., Tridimas, Y.D., Woolley, N.H., 1990. A study of turbulent flows in pipe bends. Proceedings of the Institution of Mechanical Engineers 204, 399-408.

Anwer, M., So, R.M.C., 1989. Rotation effects on a fully-developed turbulent pipe flow. Experiments in Fluids 8, 33-40.

Anwer, M., So, R.M.C., 1993. Swirling turbulent flow through a curved pipe. Part I: effect of swirl and bend curvature. Experiments in Fluids $14,85-96$.

Belleville, P., Nouri, L., Legrand, J., 1992. Mixing characteristics in the torus reactor. Chemical Engineering and Technology 15, 282-289.

Bergstrom, D.J., Bender, T., Adamopoulos, G., Postlethwaite, J., 1998. Numerical prediction of wall mass transfer rates in turbulent flow through a $90^{\circ}$ two-dimensional bend. Canadian Journal of Chemical Engineering 76, 728-737.

Chang, K.C., Hsieh, W.D., Chen, C.S., 1995. A modified low-Reynolds-number turbulence model applicable to recirculating flow in pipe expansion. Journal of Fluids Engineering 117, 417-423.

Cheng, G.C., Farokhi, S., 1992. On turbulent flows dominated by curvature effects. Journal of Fluids Engineering 114, 52-57.

Clayton, B.R., Morsi, Y.S.M., 1984. Determination of principal characteristics of turbulent swirling flow along annuli. Part 1: measurement of time mean parameters. International Journal of Heat and Fluid Flow 5 (4), 195-203.

Farias Neto, S.R., Legentilhomme, P., Legrand, J., 1998. Finite-element simulation of laminar swirling decaying flow induced by means of a tangential inlet in an annulus. Computer Methods in Applied Mechanics and Engineering 165, 189-213.

Ghia, K.N., Ghia, U., Shin, C.T., 1987. Study of fully developed incompressible flow in curved ducts using a multi-grid technique. Journal of Fluids Engineering 109, 226-236.

Gupta, A.K., Lilley, D.G., Syred, N., 1984. Swirl Flows. Abacus Press, Cambridge.

Hinze, J.O., 1975. Turbulence. McGraw-Hill, New York.

Iacovides, H., Launder, B.E., Li, H.Y., 1996a. The computation of flow development through stationary and rotating U-ducts of strong curvature. International Journal of Heat and Fluid Flow 17 (1), 22-33.
Iacovides, H., Launder, B.E., Li, H.Y., 1996b. Application of a reflection-free DSM to turbulent flow and heat transfer in a square-sectioned U-bend. Experimental Thermal and Fluid Science 13, 419-429.

Jayanti, S., Hewitt, G.F., Kightley, J.R., 1990. Fluid flow in curved ducts. International Journal for Numerical Methods in Fluids 10, 567-589.

Khalid, A., Legrand, J., 2001. Energy dissipation distribution and mixing in a torus reactor. Chemical Engineering Communications 185, 141-164.

Khalid, A., Legrand, J., Rosant, J.M., 1996. Turbulent flow induced by an impeller in a closed toroidal loop. Journal of Fluids Engineering $118,677-684$.

Kim, N., Rhode, D.L., 2000. Streamwise curvature effect on the incompressible turbulent mean velocity over curved surfaces. Journal of Fluids Engineering 122, 547-551.

Kim, J., Simon, T.W., 1988. Measurements of the turbulent transport of heat and momentum in convexly curved boundary layers: effects of curvature, recovery and free-stream turbulence. Journal of Turbomachinery $110,81-87$.

Lai, Y.G., So, R.M.C., Anwer, M., Hwang, B.C., 1991. Calculations of a curved-pipe flow using Reynolds stress closure. Proceedings of the Institution of Mechanical Engineers 205, 231-244.

Launder, B.E., Spalding, D.B., 1974. The numerical computation of turbulent flows. Computer Methods in Applied Mechanics and Engineering 3, 269-289.

Legrand, J., Guéguen, J., Berot, S., Popineau, Y., Nouri, L., 1997. Acetylation of pea isolate in a torus microreactor. Biotechnology and Bioengineering 53 (4), 410-414.

Nouri, L., Legrand, J., Popineau, Y., Belleville, P., 1997. Enzymatic hydrolysis of wheat proteins. Part II . Comparison of performance of batch-stirred and torus reactors. Chemical Engineering Journal 65, 195-199.

Parchen, R.R., Steenbergen, W., 1998. An experimental and numerical study of turbulent swirling pipe flows. Journal of Fluids Engineering $120,54-61$

Rütten, F., Meinke, M., Shröder, W., 2001. Large-eddy simulations of $90^{\circ}$ pipe bend flows. Journal of Turbomachinery 2, 1-14.

Steenbergen, W., Voskamp, J., 1998. The rate of decay of swirl in turbulent pipe flow. Flow Measurements and Instrumentation 9, 67-78.

Sudo, K., Sumida, M., Hibara, H., 1998. Experimental investigation on turbulent flow in a circular-sectioned 90-degrees bend. Experiments in Fluids 25, 42-49.

Van de Vosse, F.N., Van Steenhoven, A.A., Segal, A., Janssen, J.D., 1989. A finite element analysis of the steady laminar entrance flow in a $90^{\circ}$ curved tube. International Journal for Numerical Methods in Fluids 9, 275-287.

Wang, J., Shirazi, S., 2000. A simultaneous variable solution procedure for laminar and turbulent flows in curved channels and bends. Journal of Fluids Engineering 122, 552-559.

Wang, J., Shirazi, S., 2001. A CFD based correlation for mass transfer coefficient in elbows. International Journal of Heat and Mass Transfer 44, 1817-1822.

Wilcox, D.C., 1998. Turbulence Modeling for CFD. DCW Industries, La Canada, CA.

Wolfstein, M., 1969. The velocity and temperature distribution of one-dimensional flow with turbulence augmentation and pressure gradient. International Journal of Heat and Mass Transfer 12, 301-318. 